\title{
Cepheids and other variable stars and the distance to the Galactic Centre
}

\author{
Noriyuki Matsunaga \\ Department of Astronomy, The University of Tokyo, 7-3-1 Hongo, Bunkyo-ku, \\ Tokyo 113-0033, Japan \\ email: matsunaga@astron.s.u-tokyo.ac.jp
}

\begin{abstract}
We review and discuss results of our survey of variable stars towards the Galactic Centre and their distances. In our near-infrared monitoring survey using IRSF/SIRIUS, we detected a number of Miras and Cepheids (both classical and type II) within 20 arcmin of the Galactic Centre. These distance indicators yield a distance to the Galactic Centre of between 7.5 and $8.5 \mathrm{kpc}$. A new calibration of the red clump also leads to a distance of $\sim 8 \mathrm{kpc}$. For these indicators, which are luminosity-based, a large uncertainty resides in the correction for the foreground extinction, which depends on the reddening law. Nevertheless, our estimates are consistent with previous estimates based the kinematics of stars near the Galactic Centre, and this supports the reddening law we use.
\end{abstract}

Keywords. Cepheids, stars: variables: other, Galaxy: center, distance scale

\section{Introduction}

The distance to the Galactic Centre (GC) is a fundamental value related to Galactic structure. Whereas the IAU recommended a distance of $\mathrm{R}_{0}(\mathrm{GC})=8.5 \mathrm{kpc}$ in 1985 , estimates in many papers show a scatter between 7.5 and $9.5 \mathrm{kpc}$. A good review of previous estimates is given by Gillessen (this volume). A fraction of previous estimates rely on data from the general region of the Galactic bulge and may be affected to some extent by the bar and other structures of the bulge. Here we concentrate on results obtained in areas close to the GC.

Some types of pulsating stars follow period-luminosity relations (PLRs) which can be used as distance indicators. In particular, the PLR of classical Cepheids has been used extensively for measuring distances to external galaxies and to determine the Hubble constant (e.g., Freedman et al. 2001; Riess et al. 2009). A fundamental uncertainty resides in the controversial effect of metallicity on the PLR. Recent investigations, both theoretical and observational, suggest that the effect is negligible or at least small for the infrared (IR) PLR (Bono et al. 2010; Freedman \& Madore 2011; Storm et al. 2011).

PLRs are also found for type II Cepheids and Miras. It is well-known that the PLR of type II Cepheids is fainter than that of classical Cepheids (Sandage \& Tammann 2006). For type II Cepheids, a clear and tight PLR in the near-IR was recently established by Matsunaga et al. (2006, 2011a). Whilst a small offset may exist among the PLRs of shortperiod type II Cepheids, BL Her-type, in different environments, such an effect seems rather small for long-period type II Cepheids, W Vir-type (Matsunaga et al. 2011a). Miras are long-period pulsating stars on the asymptotic giant branch, and their PLR is tight in the IR and in bolometric magnitudes (Glass \& Lloyd Evans 1981; Feast et al. 1989). Any metallicity effect on the relation for Miras has been less well-studied compared to those affecting Cepheids, although this effect is unlikely large (Whitelock et al. 2008; this volume). 
The near-IR PLRs of these variable stars are useful to measure the distance to the GC once an appropriate data set of objects in its neighbourhood becomes available. Because of the significant foreground extinction $\left(A_{V} \geqslant 30 \mathrm{mag}\right)$, IR time-series observations are essential.

\section{The IRSF survey of variable stars near the GC}

\subsection{Observations}

We used the IRSF $1.4 \mathrm{~m}$ telescope and the SIRIUS near-IR camera for the monitoring survey (see Fig. 1). This system was established by Nagoya University and the National Astronomical Observatory of Japan. The facility is sited at the Sutherland station of the South African Astronomical Observatory (Nagashima et al. 1999; Glass \& Nagata 2000; Nagayama et al. 2003). The facility can take images in the $J H K_{\mathrm{s}}$ filters simultaneously and has a $7.7 \times 7.7 \operatorname{arcmin}^{2}$ field of view. The seeing is typically $1.3^{\prime \prime}$ and around $1^{\prime \prime}$ at best. Since the start in 2000, IRSF equipped with SIRIUS has been operating stably for more than 10 years.

We repeatedly observed the $20 \times 30 \mathrm{arcmin}^{2}$ region centred on the GC $(12 \mathrm{IRSF} / \mathrm{SIRIUS}$ fields). We carried out $\sim 90$ epochs of monitoring imaging between 2001 and 2008. Many of the images were obtained in 2005 and 2006. Each set of observations comprises ten exposures of five seconds at slightly dithered positions. The number of data sets collected per night ranges from one to six, depending on the field. The saturation limits are 9.5, 9.5 and $9.0 \mathrm{mag}$, and the detection limits are around 16.4, 14.5 and $13.1 \mathrm{mag}$ in $J, H$ and $K_{\mathrm{s}}$, respectively. The definition of these values was given in Matsunaga et al. (2009). The detection limits vary across the survey region, depending on the degree of crowding. The limits also change from frame to frame depending on the weather conditions.

\subsection{Survey results}

The IRSF/SIRIUS survey of the GC region allowed us to detect a number of variable stars: Miras, classical Cepheids, type II Cepheids and others. The data analysis and catalogue of Miras and other long-period variables are provided by Matsunaga et al. (2009), and those of Cepheids and other short-period variables by Matsunaga et al. (2011b, 2013).
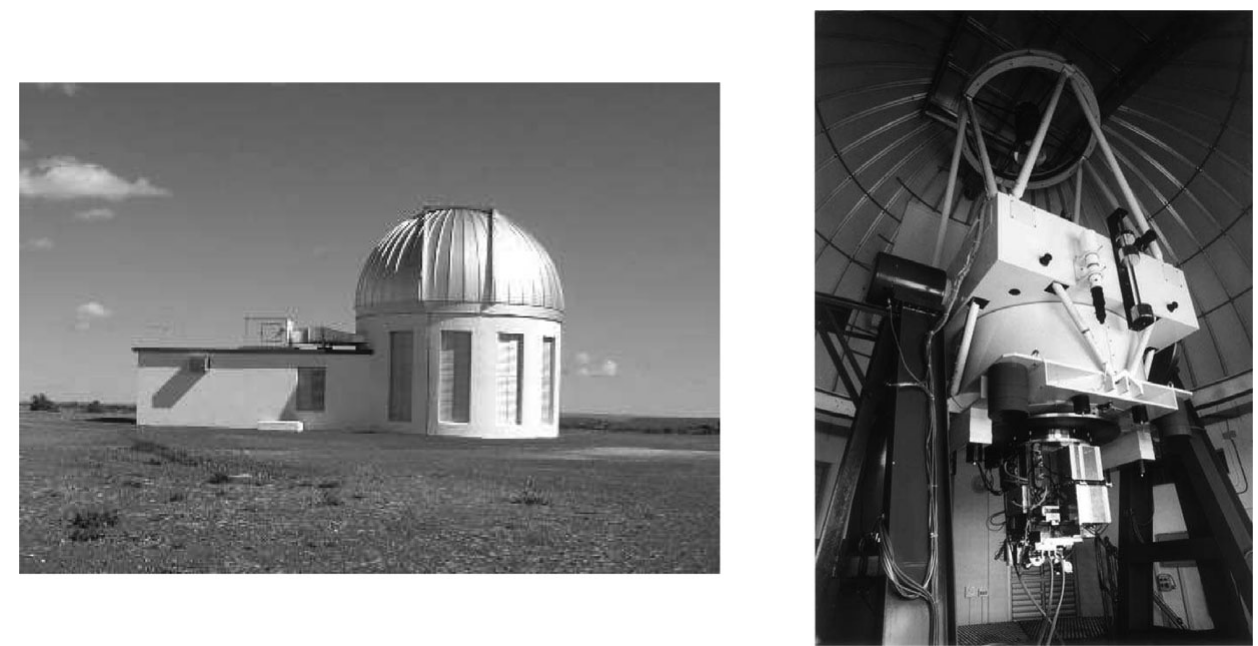

Figure 1. Infrared Survey Facility: enclosure (left) and the $1.4 \mathrm{~m}$ telescope with the SIRIUS camera attached to the Cassegrain focus (right). 
Our survey region is similar to that of a previous near-IR survey by Glass et al. (2001), who discovered more than 400 Miras. In fact, we detected most of these, as well as over 1,000 additional long-period variables. They include Miras with various periods and variable stars with less regularity and/or smaller amplitudes than Miras. In addition, three objects were identified as luminous blue variables, around the Quintuplet cluster. $\dagger$ Although the vast majority of objects in the 2009 catalogue are expected to be red giants or supergiants, some may subsequently be identified as luminous blue variables, in particular those whose light curves in our data set are indistinguishable from those of slowly pulsating irregular variables. On the other hand, pulsation periods are determined for $\sim 550$ long-period variables, and their $K_{\mathrm{s}}$-band amplitudes indicate that most of these are Miras.

No Cepheids (either classical or type II) had been found near the GC prior to our survey. We discovered three classical Cepheids (Matsunaga et al. 2011b) and 16 type II Cepheids as well as other types of short-period, $\leqslant 60$ days, variables (Matsunaga et al. 2013). The classical Cepheids are of particular interest, both for the distance scale and as regards the stellar population of the GC. They are considered to belong to the Galactic nuclear bulge, which is a disk-like distribution of young-to-old stars with a radius of $\sim 200$ pc (Launhardt et al. 2002). All classical Cepheids have periods of $\sim 20$ days, which indicates an age of $\sim 25 \mathrm{Myr}$ (Bono et al. 2005). Since they are the first tracers with an accurate age on the order of $10^{7} \mathrm{yr}$, they provide an important constraint on the stellar formation history in the nuclear bulge (Matsunaga et al. 2011b). Their existence, and kinematics and chemical features when measured, have significant implications for the evolution of the nuclear bulge and neighbouring systems (Matsunaga 2012; Bono et al. 2013).

\section{The distance to the GC}

\subsection{Variable stars}

In view of the large effects of reddening in the GC region, we consider a reddening-free PLR in $H$ and $K_{\mathrm{s}}$,

$$
W_{H K_{\mathrm{s}}}=K_{\mathrm{s}}-1.44\left(H-K_{\mathrm{s}}\right)
$$

where the coefficient is taken from the reddening law, $A_{K_{\mathrm{s}}} / E\left(H-K_{\mathrm{s}}\right)=1.44$, of Nishiyama et al. (2006a). They observed red clump giants near the GC with IRSF/SIRIUS to derive the coefficient. Fig. 2 plots $W\left(H K_{\mathrm{s}}\right)$ against the period for the variables in the GC (black points and plus symbols) and their counterparts in the Large Magellanic Cloud (LMC; grey). The assumed distance moduli, $\mu_{0}(\mathrm{GC})=14.50 \mathrm{mag}$ and $\mu_{0}(\mathrm{LMC})=18.50 \mathrm{mag}$, are temporarily subtracted for the comparison. Note that we considered Miras with periods between 100 and 350 days, which are scarcely affected by circumstellar extinction and show a tight PLR in the $K_{\mathrm{s}}$ band (Matsunaga et al. 2009; Ita \& Matsunaga 2011). A few type II Cepheids (two foreground and one with uncertain photometry) have been excluded from the plot. The good agreement between the GC objects and the LMC counterparts already indicates a GC modulus of $\sim 14.5$ mag.

As discussed and shown by Matsunaga et al. (2009), both the distance and extinction can be obtained based on PLRs in more than two filters. The distance can be equivalently derived using the $W_{H K_{\mathrm{s}}}$ relation, as long as the coefficient-1.44 in Eq. (3.1) - is taken from the same reddening law. We obtained the $\mu_{0}$ and $A\left(K_{\mathrm{s}}\right)$ values for Miras and

$\dagger$ These are the Pistol Star, qF362 and G0.120-0.048; cf. Mauerhan et al. (2010); they correspond to numbers 1207, 1248 and 1066 of Matsunaga et al. (2009). 


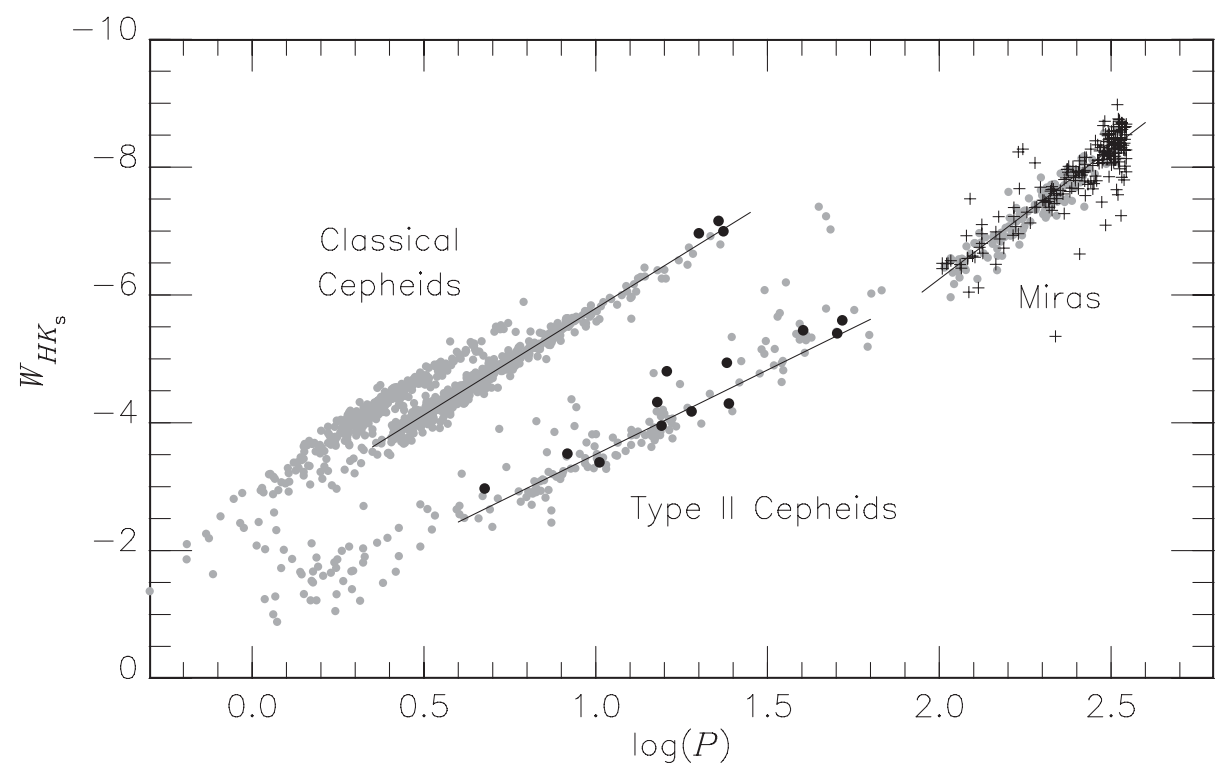

Figure 2. $\log P$ versus $W\left(H K_{\mathrm{s}}\right)$ relations for three kinds of variable stars. Grey symbols indicate LMC objects, and black dots and plus symbols indicate Cepheids and Miras, respectively, near the GC. Shifts are added based on the approximate distance moduli of the LMC (18.50 mag) and the Galactic bulge $(14.50 \mathrm{mag})$. Linear fits are shown for the LMC objects, taken from Matsunaga et al. (2009, 2011a).

Table 1. Distance to the Galactic Centre derived using several indicators. The $\mu_{0}$ values are listed as well as the statistical and systematic errors and the numbers of objects used.

\begin{tabular}{lcccr}
\hline Type & $\begin{array}{c}\mu_{0} \\
(\mathrm{mag})\end{array}$ & $\begin{array}{c}\text { Stat. Error } \\
(\mathrm{mag})\end{array}$ & $\begin{array}{c}\text { Syst. Error } \\
(\mathrm{mag})\end{array}$ & $N$ \\
\hline Classical Cepheids & 14.49 & 0.08 & 0.09 & 3 \\
Type II Cepheids & 14.38 & 0.13 & 0.11 & 5 \\
Miras & 14.63 & 0.02 & 0.11 & 100 \\
Red clump & 14.53 & 0.03 & 0.10 & - \\
\hline
\end{tabular}

Cepheids in Matsunaga et al. (2009, 2011a, 2013). The distribution and the extinction values of variable stars are presented in Fig. 3. Circles of various sizes are scattered across our survey region, which indicates that the foreground extinction varies in a complicated manner as function of the line of sight.

Table 1 lists averages of the distance estimates as well as the relevant errors. The main sources of systematic errors are uncertainties in the reddening law, the LMC distance and the possible metallicity effect on the PLRs. The errors in the reddening law $( \pm 0.07 \mathrm{mag})$ and the LMC distance $( \pm 0.05 \mathrm{mag})$ have a common effect on the three distance indicators. In contrast, metallicity effects on the PLRs may be different for the three relations and, reflecting different levels of previous effort, the remaining errors are also different. As mentioned in Section 1, a number of previous studies suggest that any metallicity effect on the near-IR PLR of classical Cepheids is negligible $( \pm 0.03 \mathrm{mag})$, while the effect on the PLR of Miras is not well constrained $( \pm 0.07 \mathrm{mag})$. The results of Matsunaga et al. (2006, 2011a) suggest that any population effect on the PLR of type II Cepheids (W Vir stars) is small. Nevertheless, to be conservative we adopt an uncertainty of 0.07 mag for this effect. 


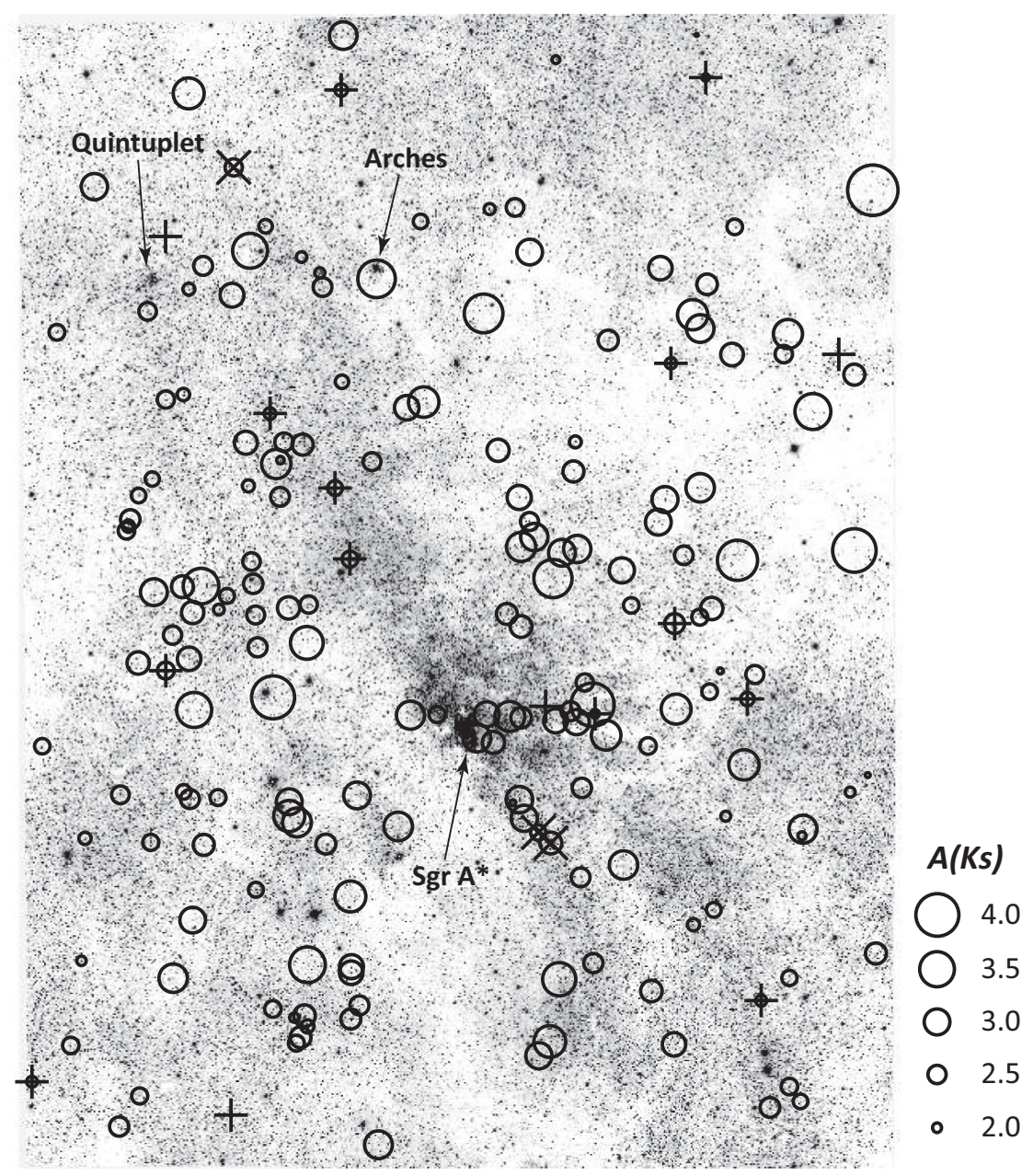

Figure 3. Field of the IRSF/SIRIUS survey $\left(20 \times 30 \operatorname{arcmin}^{2}\right)$ and distribution of variable stars with known distances. The extinction obtained for each object is indicated by the size of the circle (legend shown next to the panel). Cross and plus symbols are overlaid for classical and type II Cepheids, respectively. The positions of Sgr A*, the Arches and Quintuplet clusters are indicated.

\subsection{Red clump}

Nishiyama et al. $(2006 \mathrm{~b})$ carried out $J H K_{\mathrm{s}}$ observations of red clump stars in the region centred on the GC using IRSF/SIRIUS. Recently, Laney et al. (2012) obtained new high-precision $J H K_{\mathrm{s}}$ magnitudes of red clump giants with Hipparcos parallaxes, which yield a new calibration of the red clump. Whereas Nishiyama et al. (2006b) adopted $\left(H-K_{\mathrm{s}}\right)_{0}=0.07 \mathrm{mag}$ and $K_{\mathrm{s}}=-1.59 \mathrm{mag}$ based on the theoretical isochrones of Bonatto et al. (2004), Laney et al. (2012) obtained $\left(H-K_{\mathrm{s}}\right)_{0}=0.123 \mathrm{mag}$ and $K_{\mathrm{s}}=-1.613$ mag. This new calibration leads to $\mu_{0}(\mathrm{GC})=14.53 \pm 0.10 \mathrm{mag}$, without any population effect taken into account. There is a large scatter in metallicities of red clump giants in the bulge and the median metallicity seems slightly higher than solar abundance (Hill et al. 2011). The error, adopted from Nishiyama et al. (2006b), includes the uncertainty owing to a possible population effect. 


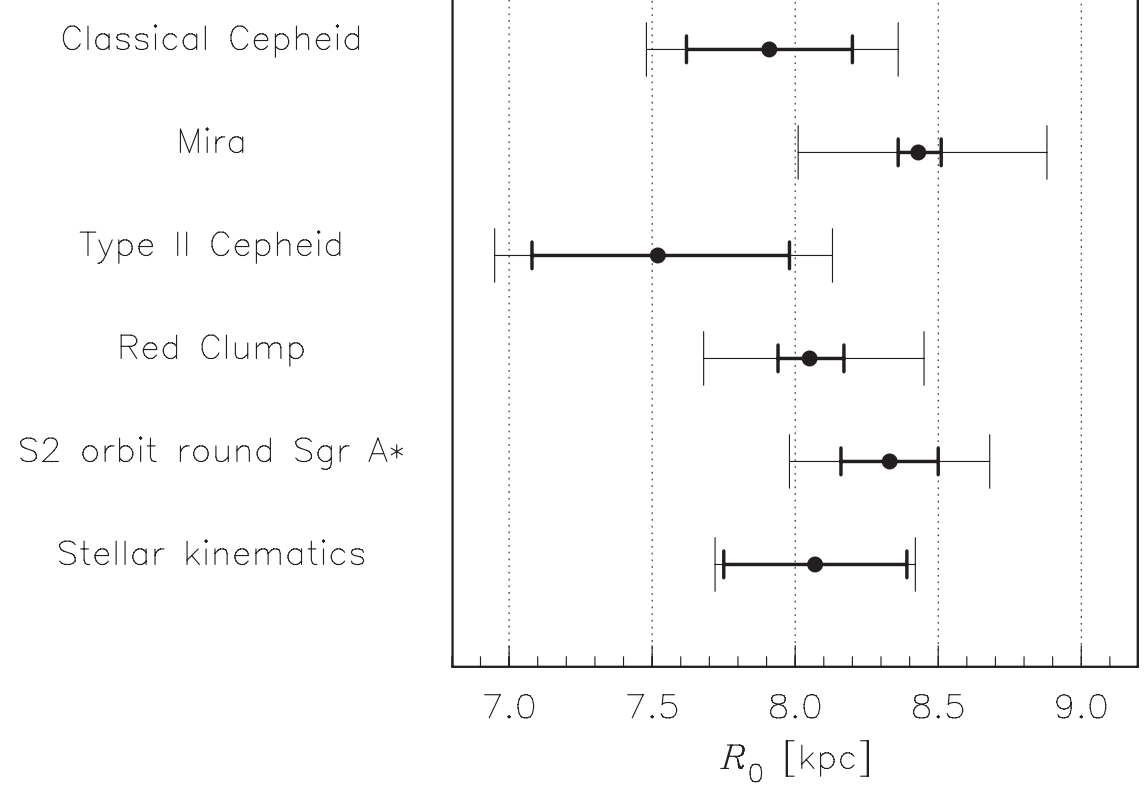

Figure 4. Estimates of the distance $\mathrm{R}_{0}$ to the GC based on various methods (see text for references). The larger error bars indicate both statistical and systematic errors, whereas the thick bars only consider the statistical errors.

\subsection{Comparison with various distance indicators}

The above estimates based on near-IR data of stellar distance indicators are compared with results from kinematic methods in Fig. 4. The latter methods are the fits of Keplerian rotation of the star S2 around Sgr A* (Gillessen et al. 2009) and the statistical-parallax method applied to the central stellar cluster (Trippe et al. 2008). Although statistical errors are rather small for some kinds of indicators, significant systematic errors $( \pm 0.4 \mathrm{kpc})$ remain. Within these uncertainties, the photometric and kinematic determinations are in satisfactory agreement and indicate a value of $\mathrm{R}_{0}$ close to $8.0 \mathrm{kpc}$.

While metallicity effects on stellar distance indicators are important for many distancescale problems, the uncertainties in the distance modulus of LMC objects (or calibrators) and the reddening law are also significant in our case. Among the latter two, the LMC modulus has been investigated using many methods, and we expect more accurate estimates which can be cross-checked with each other (see, for example, Pietrzyński et al., this volume). On the other hand, determination of the error in the foreground extinction towards the GC is a challenging problem. As long as luminosity-based distance indicators are considered, the effects of foreground extinction are more or less the same for GC objects. In addition, the reddening law may vary from place to place to some extent (Zasowski et al. 2009), and the analysis should focus on the GC region. In this regard, the agreement of the photometric and kinematic results provides an important constraint on the reddening law. We adopted the reddening law of Nishiyama et al. (2006a) and, for instance, this gives $A_{K_{\mathrm{s}}}=2.5 \mathrm{mag}$ for a typical value of $E\left(H-K_{\mathrm{s}}\right)=1.8 \mathrm{mag}$. In contrast, the reddening law of Rieke \& Lebofsky (1985) gives 3.2 mag and would lead to an unacceptably small value of $\mathrm{R}_{0}(\mathrm{GC})$. Our result suggests that the reddening law towards the GC should not be too different from Nishiyama's law. 


\section{Acknowledgements}

This article is partly based on recent publications in collaboration with Takahiro Kawadu, Shogo Nishiyama, Takahiro Nagayama, Hirofumi Hatano, Motohide Tamura, Naoto Kobayashi, Giuseppe Bono, Ian S. Glass, Michael W. Feast and Tetsuya Nagata. Especially, I am grateful to Michael and Giuseppe for discussions on the distance scale and stellar populations, and for useful comments on this manuscript. I also thank Masuo Tanaka for a discussion on luminous blue variables. The author acknowledges support from a Grant-in-Aid for Research Activity Start-up (No. 22840008) and a Grant-in-Aid for Young Scientists (No. 80580208) from the Japan Society for the Promotion of Science (JSPS).

\section{References}

Bonatto, C., Bica, E., \& Girardi, L. 2004, A\&A, 415, 571

Bono, G., Marconi, M., Cassisi, S., Caputo, F., Gieren, W., \& Pietrzyński, G. 2005, ApJ, 621, 966

Bono, G., Caputo, F., Marconi, M., \& Musella, I. 2010, ApJ, 715, 277

Bono, G., Matsunaga, N., Inno, L., Lagioia, L., \& Genovali, K. 2012, in: Sant Cugat Forum on Astrophysics (Torres, D. F., \& Reimer, O., eds.), in press

Feast, M. W., Glass, I. S., Whitelock, P. A., \& Catchpole, R. M. 1989, MNRAS, 241, 375

Freedman, W. L. \& Madore, B. F. 2011, ApJ, 734, 46

Freedman, W. L., et al. 2001, ApJ, 553, 47

Gillessen, S., Eisenhauer, F., Fritz, T. K., Bartko, H., Dodds-Eden, K., Pfuhl, O., Ott, T., \& Genzel, R. 2009, ApJ, 707, L114

Glass, I. S. \& Lloyd Evans, T. 1981, Nature, 291, 303

Glass, I. S., Matsumoto, S., Carter, B. S., \& Sekiguchi, K. 2001, MNRAS, 321, 77

Glass, I. S. \& Nagata, T. 2000, Mon. Not. Astron. Soc. S. Africa, 59, 110

Hill, V., Lecureur, A., Gómez, A., et al. 2011, A\& A, 534, 80

Ita, Y. \& Matsunaga, N. 2011, MNRAS, 412, 2345

Launhardt, R., Zylka, R., \& Mezger, P. G. 2002, A\&A, 384, 112

Laney, C. D., Joner, M. D., \& Pietrzyński, G. 2012, MNRAS, 419, 1634

Matsunaga, N., Fukushi, H., Nakada, Y., et al. 2006, MNRAS, 370, 1979

Matsunaga, N., Kawadu, T., Nishiyama, S., et al. 2009, MNRAS, 399, 1709

Matsunaga, N., Feast, M. W., \& Soszyński, I. 2011a, MNRAS, 413, 223

Matsunaga, N., Kawadu, T., Nishiyama, S., et al. 2011b, Nature, 477, 188

Matsunaga, N. 2012, J. Phys. Conf. Ser., 372, 12026

Matsunaga, N., Feast, M. W., Kawadu, T., et al. 2013, MNRAS, in press (arXiv:1211.0151)

Mauerhan, J. C., Morris, M. R., Cotera, A., Dong, H., Wang, Q. D., Stolovy, S. R., Lang, C., \& Glass, I. S. 2010, ApJ, 713, L33

Nagashima, C., Nagayama, T., Nakajima, Y., et al. 1999, in: Proc. Star Formation (Nakamoto, T., ed.), p. 397

Nagayama, T., Nagashima, C., Nakajima, Y., et al. 2003, Proc. SPIE, 4841, 459

Nishiyama, S., Nagata, T., Kusakabe, N., et al. 2006a, ApJ, 638, 839

Nishiyama, S., Nagata, T., Sato, S., et al. 2006b, ApJ, 647, 1093

Rieke, G. H. \& Lebofsky, M. J. 1985, ApJ, 288, 618

Riess, A. G., Macri, L., Li, W., et al. 2009, ApJS, 183, 109

Sandage, A. \& Tammann, G. A. 2006, ARA\&A, 44, 93

Storm, J., Gieren, W., Fouqué, P., Barnes, T. G., Soszyński, I., Pietrzyński, G., Nardetto, N., \& Queloz, D. 2011, Aछ A, 534, 95

Trippe, S., Gillessen, S., Gerhard, O. E., et al. 2008, A\&A, 492, 419

Whitelock, P. A., Feast, M. W., \& van Leeuwen, F. 2008, MNRAS, 386, 313

Zasowski, G., Majewski, S. R., Indebetouw, R., et al. 2009, ApJ, 707, 510 